\title{
Enhanced electric conductivity at ferroelectric vortex cores in $\mathrm{BiFeO}_{3}$
}

\author{
Nina Balke ${ }^{1 \star}$, Benjamin Winchester ${ }^{2}$, Wei Ren ${ }^{3}$, Ying Hao Chu ${ }^{4,5}$, Anna N. Morozovska ${ }^{6}$, \\ Eugene A. Eliseev ${ }^{7}$, Mark Huijben ${ }^{8}$, Rama K. Vasudevan ${ }^{9}$, Petro Maksymovych ${ }^{1}$, Jason Britson ${ }^{2}$, \\ Stephen Jesse ${ }^{1}$, Igor Kornev ${ }^{10}$, Ramamoorthy Ramesh ${ }^{5}$, Laurent Bellaiche ${ }^{3}$, Long Qing Chen ${ }^{2}$ \\ and Sergei V. Kalinin ${ }^{1 \star}$
}

Topological defects in ferroic materials are attracting much attention both as a playground of unique physical phenomena and for potential applications in reconfigurable electronic devices. Here, we explore electronic transport at artificially created ferroelectric vortices in $\mathrm{BiFeO}_{3}$ thin films. The creation of one-dimensional conductive channels activated at voltages as low as $1 \mathrm{~V}$ is demonstrated. We study the electronic as well as the static and dynamic polarization structure of several topological defects using a combination of first-principles and phase-field modelling. The modelling predicts that the core structure can undergo a reversible transformation into a metastable twist structure, extending charged domain walls segments through the film thickness. The vortex core is therefore a dynamic conductor controlled by the coupled response of polarization and electron-mobile-vacancy subsystems with external bias. This controlled creation of conductive one-dimensional channels suggests a pathway for the design and implementation of integrated oxide electronic devices based on domain patterning.

T opological defects ${ }^{1}$ in condensed matter offer a powerful paradigm for nanoscale-device engineering owing to the combination of unique physical properties and the capability for their manipulation by external magnetic, electric, or strain fields without disruption of the host lattice ${ }^{2}$. Examples include vortices in superconductors ${ }^{3}$, defects in topological insulators ${ }^{4}$, and domain walls in ferroics. The numerous examples of novel functionalities at the domain walls enabled by stabilization of a high-symmetry phase and order-parameter coupling ${ }^{5}$ include domain wall ferroelectricity in paraelectric materials ${ }^{6-8}$, enhanced electronic conductivity ${ }^{9-11}$, magnetoelectric coupling ${ }^{12}$, magnetic phase transitions ${ }^{13}$, and ionic phenomena ${ }^{14}$. This potentially facilitates a broad spectrum of reconfigurable magnetoelectric, optoelectonic, and strain-coupled memory and logic devices through domain engineering.

All experimental and virtually all theoretical studies so far explore the properties of $2 \mathrm{D}$ topological defects, such as ferroic domain walls. At the same time, implementation in electronic devices requires the capability to create and modify $1 \mathrm{D}$ conductive objects, effectively creating 'wiring' in functional devices. The existence of 1D topological defects, namely vortices, have been reported in ferroelectric systems and has led to a number of experimental ${ }^{15-18}$ and theoretical efforts ${ }^{19-23}$ aimed at visualizing these defects and probing their properties. Most recent studies confirm the existence of vortices or flux-closure domains in ferroelectrics through direct imaging using transmission electron microscopy ${ }^{24-26}$. In all these reports, the vortices have been observed in ferroelectric nanostructures, in as-grown thin films or single crystals. Here, we report on the controlled creation and probing of the electronic properties of 1D topological defects. Specifically, we explore the physical properties of artificially engineered domain junctions forming vortex or antivortex states in $50 \mathrm{~nm}$ (001)oriented multiferroic $\mathrm{BiFeO}_{3}$ (BFO) thin films by local current detection using scanning probe microscopy (SPM), and decipher the associated mesoscopic and atomistic conduction mechanisms to establish the origins of vortex conduction.

\section{Creation and transport measurements of vortex structures} In this study, the vortex state denotes the centre of a closure domain formed by the in-plane polarization components of four ferroelectric domains which are aligned head-to-tail, and can formally be described by a planar spin configuration with a winding number of +1 . The antivortex is defined as a domain arrangement where the in-plane polarization components point towards and away from each other, forming a point at the four-domain junction (winding number -1). Here, vortex domain patterns were created by applying $-9 \mathrm{~V}$ to the SPM tip during a specific tip motion, as described previously ${ }^{27}$. A negative switching voltage was chosen to switch the out-of-plane domain orientation from down to up to eliminate the displacement current contribution from out-of-plane domain switching ${ }^{28}$. Subsequent current measurements were

\footnotetext{
${ }^{1}$ The Center for Nanophase Materials Sciences, Oak Ridge National Laboratory, Oak Ridge, Tennessee 37831, USA, ${ }^{2}$ Department of Materials Science and Engineering, Pennsylvania State University, University Park, Pennsylvania 16802, USA, ${ }^{3}$ Physics Department and Institute for Nanoscience and Engineering, University of Arkansas, Fayetteville, Arkansas 72701, USA, ${ }^{4}$ Department of Materials Science and Engineering, National Chiao Tung University, Hsinchu 30010, Taiwan, ${ }^{5}$ Department of Materials Science and Engineering and Department of Physics, University of California, Berkeley, California 94720, USA, ${ }^{6}$ Institute for Problems of Materials Science, National Academy of Science of Ukraine, UA-03142 Kiev, Ukraine, ${ }^{7}$ Institute of Semiconductor Physics, National Academy of Science of Ukraine, UA-03028 Kiev, Ukraine, ${ }^{8}$ Faculty of Science and Technology, MESA ${ }^{+}$Institute for Nanotechnology, University of Twente, PO Box 217, 7500 AE, Enschede, The Netherlands, ${ }^{9}$ School of Materials Science and Engineering, University of New South Wales, Sydney, New South Wales 2052, Australia, ${ }^{10}$ Laboratoire Structures, Proprietes et Modelisation des Solides, Ecole Centrale Paris, CNRS-UMR8580, Grande Voie des Vignes, 92295 Chatenay-Malabry Cedex, France. *e-mail: n2b@ornl.gov; sergei2@ornl.gov.
} 


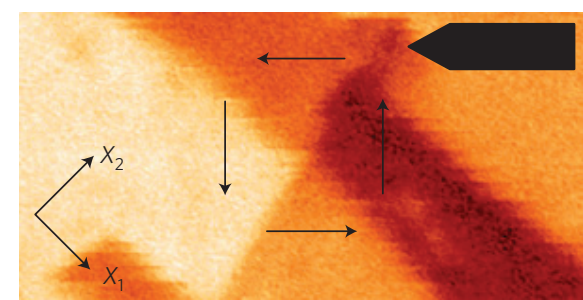

b

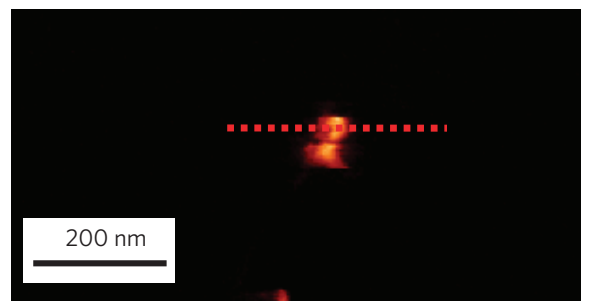

C
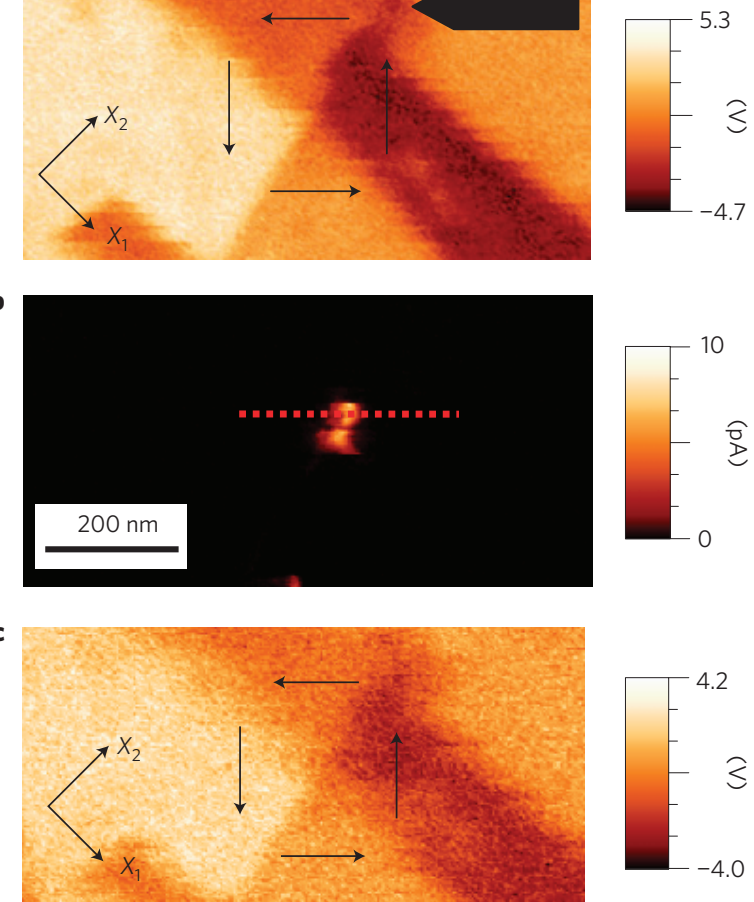

10

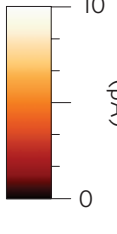

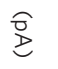

e

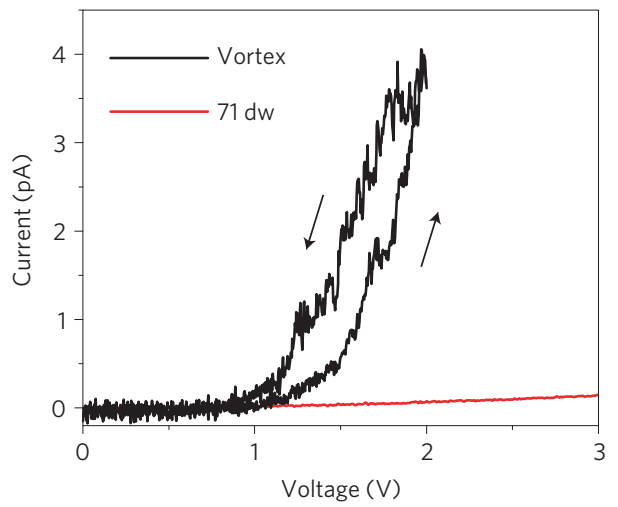

Figure 1 | Electronic properties of topological defects in ferroelectrics. $\mathbf{a}$, In-plane PFM image of vortex domain structure. $\mathbf{b}$, c-AFM image at $2 V_{d c}$,

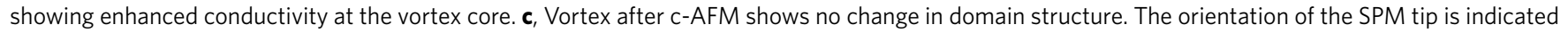
in $\mathbf{a}$ and points along the [110] direction. $\mathbf{d}$, Extracted line from the c-AFM image indicated in $\mathbf{b}$. e, $I-V$ curves measured with a stationary tip in the vortex centre (black) and at a $71^{\circ}$ domain wall (red).

performed with positive voltages applied to the bottom electrode. The topological defects were created along a line between two different stripe-like domain patterns and consisted of an alternating arrangement of vortex and antivortex domain structures. The in-plane piezoresponse force microscopy (PFM) images of a vortex domain structure are shown in Fig. 1a. The indicated domain orientations were unambiguously established by in-plane PFM imaging after $90^{\circ}$ sample rotation (see Supplementary Information).

To characterize the local electronic properties of the vortex, we employed conductive atomic force microscopy (c-AFM) imaging and single-point $I-V$ measurements. Figure $1 \mathrm{~b}$ shows the c-AFM image recorded at $2 \mathrm{~V}_{\mathrm{dc}}$ bias immediately after vortex formation. Note that the vortex core exhibits significant conductivity compared with the surrounding surface. The in-plane PFM image after c-AFM in Fig. 1c confirms that the domain pattern was not irreversibly changed during the current measurement. We further note that application of higher voltages often leads to reconstruction of the domain structure (despite the fact that the field is applied nominally in the non-switching direction), either in the vicinity of a topological defect, or over the mesoscopic region of the sample surface. Figure 1d shows a line scan through the current maximum in the vortex core. After the c-AFM image, local $I-V$ curves were measured with a stationary tip placed over the vortex core. The voltage was swept between 0 and $2 \mathrm{~V}$ (applied to the bottom electrode) at a rate of $1 \mathrm{~V} \mathrm{~s}^{-1}$ and shows a strong current increase for these low voltages (Fig. 1e). In comparison, if the $I-V$ curves are measured on one of the $71^{\circ}$ domain walls surrounding the vortex, the measured current is much smaller, as shown in Fig. 1e. Notably, the $I-V$ curve at the vortex core exhibits significant hysteresis and visible conductivity jumps. Thus, the four domain junctions exhibit a higher conductivity, with significant conduction observed above $\sim 1 \mathrm{~V}$ bias, as compared with $>3 \mathrm{~V}$ at the domain wall. The $I-V$ curve shown is reproducible if the voltages are kept low $(<2 \mathrm{~V})$ and has been measured at several vortex and antivortex cores. There does not seem to be a different conduction behaviour for vortex or antivortex domain configurations.

Application of higher bias $(>2 \mathrm{~V})$ during $\mathrm{c}-\mathrm{AFM}$ or $I-V$ measurements often leads to a reconstruction of domain structure. As an example, Fig. 2a shows the in-plane PFM image of an antivortex after application of higher bias, clearly demonstrating the induced domain twist at the topological defect. In this case, the c-AFM image measured with $2 \mathrm{~V}_{\mathrm{dc}}$ shows enhanced conductivity as well (Fig. 2b,d). Note that the measured current is of the same order of magnitude as the one measured for the vortex structure in Fig. 1. Interestingly, the antivortex is restored after the c-AFM image (Fig. 2c) and a second c-AFM scan at $2 \mathrm{~V}_{\mathrm{dc}}$ does not show any enhanced conductivity at the core. The observations from Figs 1 and 2 suggest that the localized conductivity at the vortex and antivortex cores arises from a unique response of the polarization structure at the vortex core to the applied electric field. For lower voltages, this response is reversible, whereas at higher voltages a static domain structure reconstruction is induced. The small remnant reconstructions (for intermediate pulses) can be reversed by low voltage pulses of opposite polarity. At the same time, higher voltages result in large-scale changes of domain structure that can generally not be reversed by a bias pulse and require fabrication of another vortex (Supplementary Information).

\section{High-resolution imaging of vortex structures}

To obtain further insight into the twisted domain structure and the possible origin of enhanced conductivity, we employed highresolution PFM imaging using band excitation resonance-enhanced in-plane and out-of-plane PFM (ref. 29). The flexural (out-ofplane) and torsional (in-plane) contact resonance frequencies of the 
a
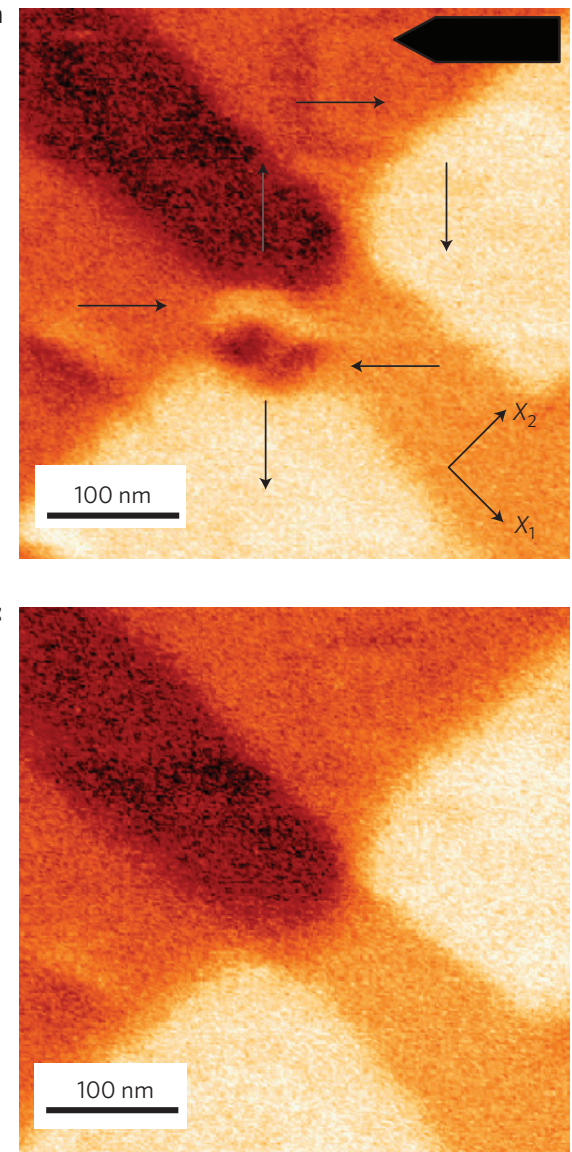

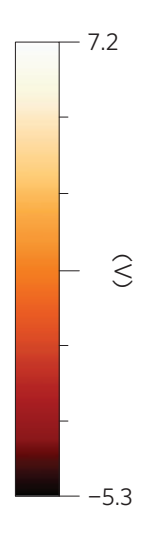

b

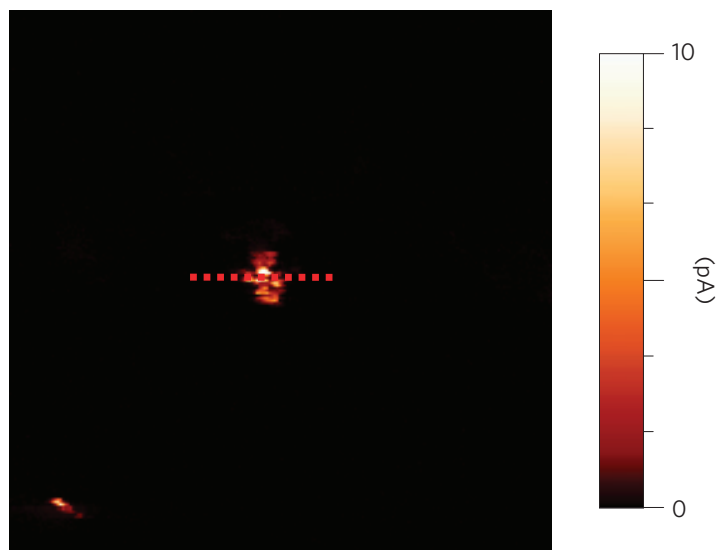

d

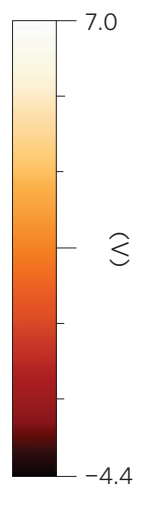

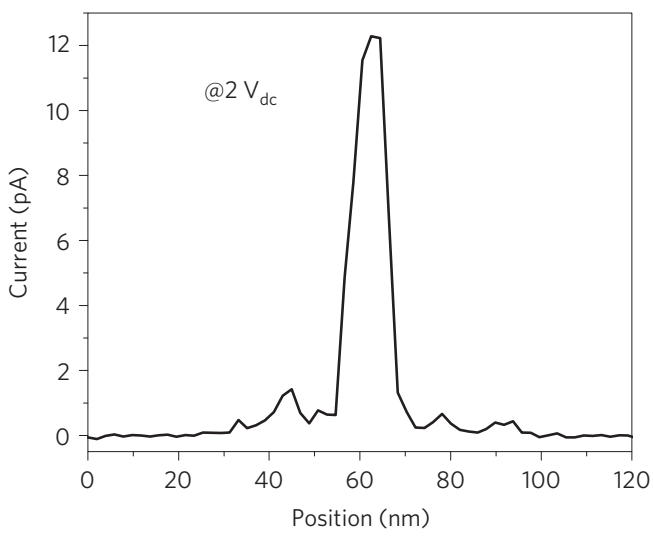

Figure 2 | Changes of the domain structure during measurement of the electronic properties of topological defects. a, In-plane PFM image showing a domain twist at an antivortex core. b, c-AFM image measured at $2 \mathrm{~V}_{\mathrm{dc}}$ showing enhanced conductivity. c, In-plane PFM image after c-AFM shows a

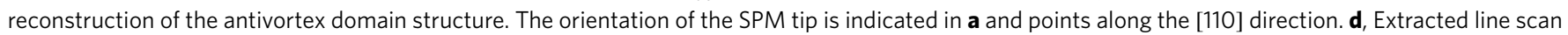
from $\mathbf{b}$ along the indicated line.

cantilever were determined as $350 \mathrm{kHz}$ and $700 \mathrm{kHz}$, respectively. Figure 3a,b shows the conventional in-plane PFM images (mixed PFM signal) measured at $30 \mathrm{kHz}$ before and after a domain twist was induced at a vortex core. The on-resonance PFM images have the advantage of higher signal-to-noise ratios and are shown in Fig. 3c-f, separated into PFM amplitude and phase images for inplane and out-of-plane. The on-resonance in-plane PFM amplitude (Fig. 3c) clearly shows the bending of the domain wall at the twist and the in-plane PFM phase (Fig. 3d) shows that the domain twist consists of in-plane polarization components that point towards each other, thus forming charged domain walls. The out-of-plane PFM amplitude and phase close to the resonance are shown in Fig. 3e,f. The out-of-plane PFM phase image reveals that the out-of-plane polarization component has switched from up (dark) to down (bright). This effect is barely visible in the conventional out-of-plane PFM image recorded at $30 \mathrm{kHz}$. This feature has been observed for other induced domain twists as well. Note the electrical fields used to perform c-AFM and $I-V$ curves are pointing upwards, thus, the remnant out-of-plane polarization component switches against the externally applied field once in the field-off state. This behaviour is reminiscent of the domain back-switching in PFM writing experiments ${ }^{30,31}$ and suggests dynamics controlled by relaxing surface or bulk screening charges, as discussed below.

\section{Electronic structure of vortex cores}

To explore the structural and electronic properties at the vortex core, their behaviour was studied using a combination of first-principles methods and phase-field modelling. Effective
Hamiltonian Monte Carlo simulations of vortex states in BFO nanodots $^{32}$ predict the distinct reduction of electric dipoles and oxygen octahedra tilt at the vortex centre compared with bulk BFO, with the magnitude of electric dipoles and oxygen octahedra tilts decreased by $7 \%$ and $2 \%$, respectively, compared with the bulk. To determine the effect of these structural changes on electronic structure, the density of states (DOS) of bulk BFO was calculated within density functional theory (DFT) for three different cases. Case (1) corresponds to the ground-state $R 3 c$ structure of BFO, as predicted by the effective Hamiltonian. Case (2) differs from case (1) by reducing the magnitude of the electric dipoles and oxygen octahedra tilts by $7 \%$ and $2 \%$, respectively. Finally, case (3) corresponds to the cubic $P m \overline{3} m$ ideal structure (for which neither polarization nor oxygen octahedral tilting exist). These DOS are shown in Fig. 4 and show a downward shift of the conduction band edge by about $0.173 \mathrm{eV}$ when going from case (1) to case (2), indicating the predicted decrease of magnitude of the electric dipoles and oxygen octahedra tilting near the vortex core is not sufficient to explain the experimentally enhanced conductivity. Interestingly, Fig. 4 also reveals that a significant decrease in the bandgap (by nearly $1.6 \mathrm{eV}$ ) is predicted to occur when going from the $R 3 c$ ground-state structure to the cubic paraelectric state of $\mathrm{BFO}$, that is, when polarization and oxygen octahedral tilting vanish $^{33}$. To establish the relative roles of the dipole moments and oxygen tilts towards bandgap modification, further calculations were performed wherein these contributions were investigated independently in the original $\mathrm{BFO}$ rhombohedral $R 3 c$ structure. Both cases lead to a bandgap reduction, but the structure without 
a

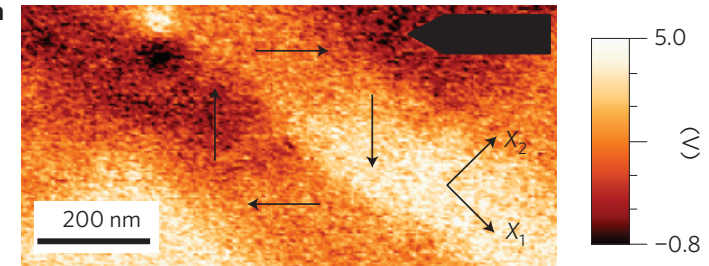

c

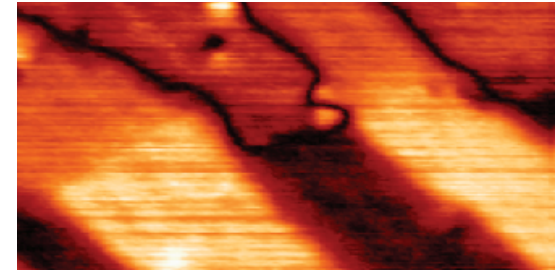

e

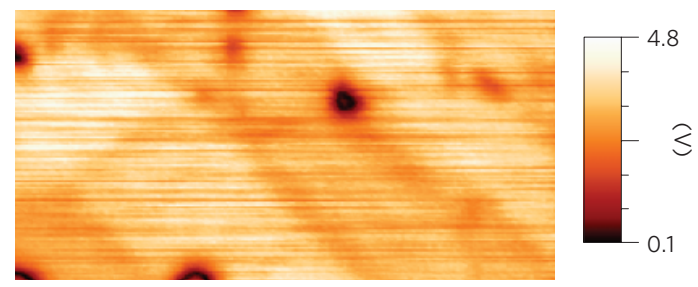

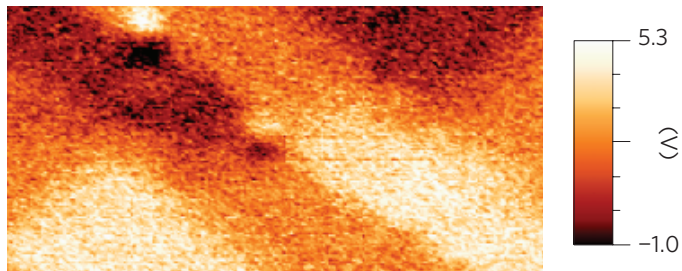

d

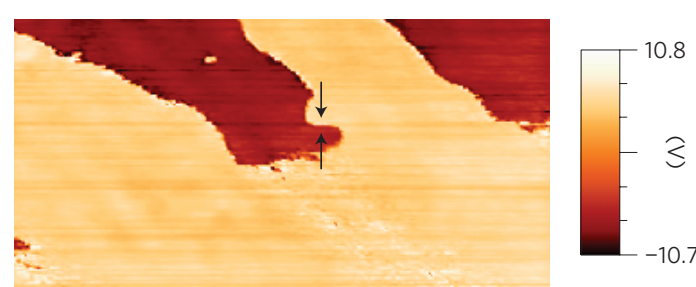

$f$

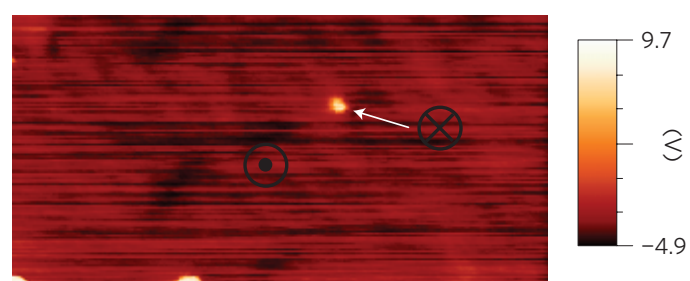

Figure 3 | High resolution imaging of the observed domain wall twist. a, Conventional in-plane PFM image of vortex domain structure. $\mathbf{b}$, Vortex with induced domain twist. c,d, In-plane PFM amplitude and phase measured at high frequencies close to the in-plane contact resonance of the cantilever. e,f, Out-of-plane PFM amplitude and phase measured at high frequencies close to the out-of-plane contact resonance of the cantilever. The orientation of the SPM tip is indicated in a and points along the [110] direction.

oxygen octahedra tilts had a narrower bandgap $(1.17 \mathrm{eV})$ than the structure without dipole moments $(1.42 \mathrm{eV}$ ) (in comparison $R 3 c$ BFO has a band-gap of $1.906 \mathrm{eV}$ ). We further note that these symmetry-induced effects are also likely to be active around other topological defects.

\section{Phase-field modelling of topological defects}

To explore the mesoscopic phenomena at the vortex core, the polarization, electric and elastic field distribution at the static vortex core and their evolution during switching were studied using phase-field modelling. The static domain structure and associated electric field, potential, and strain are shown in Fig. 5. Note the vortex core is associated with significant tensile strain, as expected for a disclination centre, and has a high electrostatic potential. The electric field trace $\left(\Sigma E_{i}\right)$, strain $\left(\Sigma s_{i i}\right)$ and stress $\left(\Sigma \sigma_{i i}\right)$ tensor traces, volume expansion energy $\left(\tilde{\beta}_{j k}^{V} \sigma_{j k}\right)$ and potential on different defect types (vortex, antivortex, domain wall, and domain) are summarized in Table 1. The evolution of these parameters with applied tip bias is summarized in Supplementary Information. In particular, we note that strain at the vortex core is almost independent of bias. We note that very similar values can be obtained by assuming zero polarization at the vortex core, $u_{i j}(r) \sim Q_{i j k l}\left(P_{k}(r) P_{l}(r)-P_{k}^{S} P_{l}^{S}\right)$, where $u_{22}^{\max }=Q_{12} P_{S}^{2} \sim-0.016$ and $Q_{11} \sim 0.032 \mathrm{~m}^{4} \mathrm{C}^{-2}, Q_{12} \sim-0.016 \mathrm{~m}^{4} \mathrm{C}^{-2}$ is the electrostriction tensor, $P_{k}(r)$ is the polarization vector, and $P_{k}^{S} \sim 1 \mathrm{C} \mathrm{m}^{-2}$ is the spontaneous polarization far from the BFO domain wall.

The phase-field results allow us to estimate the extent of electron, hole, and vacancy accumulation at the topological defects. In this, we note that phase-field results provide the lowest energy state achieved through polarization relaxation only for defined electrical and mechanical boundary conditions. In the presence of electron and vacancy mobility, the vacancy/electron accumulation will further change the concentration and electroelastic field distributions, necessitating self-consistent modelling. However, the tendency for preferred segregation and spatial geometry of active regions can be estimated from the phase-field data.
To estimate the oxygen vacancy segregation and electron/hole accumulation, we note that in thermodynamic equilibrium $N_{V}^{+}(r)=N_{V 0}^{+} \exp \left(\left(\tilde{\beta}_{j k}^{V} \sigma_{j k}(r) / \rho-e \varphi(r)\right) / k_{\mathrm{B}} T\right)$ and $n(r)=n_{0}$ $\exp \left(e \varphi(r) / k_{\mathrm{B}} T\right)$, where $k_{\mathrm{B}}=1.3807 \times 10^{-23} \mathrm{~J} \mathrm{~K}^{-1}, T$ is the absolute temperature, $\varphi(r)$ is the electric potential, $e$ is elementary charge, $\sigma_{k l}(r)$ is the stress tensor, $\tilde{\beta}_{i j}^{V} \approx 0.23$ is the Vegard expansion stress tensor for vacancies, $\rho=8.055 \times 10^{28}$ is the number of atoms per unit volume in the material, and $N_{V 0}^{+}$is the vacancy concentration far from the ferroelectric/ferroelastic wall. From these estimates, the vacancy accumulation at the vortex core, $N_{V}^{+}(r) / N_{V 0}^{+}=\exp \left(E_{S}(r) / k_{\mathrm{B}} T\right)$, where $E_{S}(r)=\tilde{\beta}_{j k}^{V} \sigma_{j k}(r) / \rho-e \varphi(r)$, can be $\gg 10^{4}$, with the primary contribution stemming from electrostatic free energy and compositional strain (see Supplementary Information). We note that the vortex core (on average) shows a strong propensity for vacancy segregation driven by elastic fields. At the same time, antivortices are prone to hole segregation. Hence, the static conductive properties of vortices and antivortices are expected to be different.

\section{Dynamic polarization behaviour at vortex cores}

We further explored the evolution of domain structures and potential and electroelastic fields at the defect cores with the probe bias, as illustrated in Fig. 6. Note that for the vortex and domain wall the dominant mode of evolution was the twist of the in-plane polarization component, in agreement with previous studies $^{27}$. For the antivortex, domain wall twists dominate at lower bias, whereas at sufficiently high bias the evolution becomes more complex. The switching dynamics can be parameterized by the voltage dependence of the average (through thickness) polarization, as shown in Fig. 6e. The strain evolution in Fig. $6 \mathrm{f}$ suggests that the disclination centre at the vortex core remains almost invariant with respect to bias. Also note that whereas electric potential can be screened by motion of low-mobility electrons and holes along the deep trap levels, the elastic fields can be screened only by vacancy redistribution. Finally, Fig. $6 \mathrm{~g}$ illustrates the evolution of the average potential as a function of tip bias. The low effective susceptibilities 
a

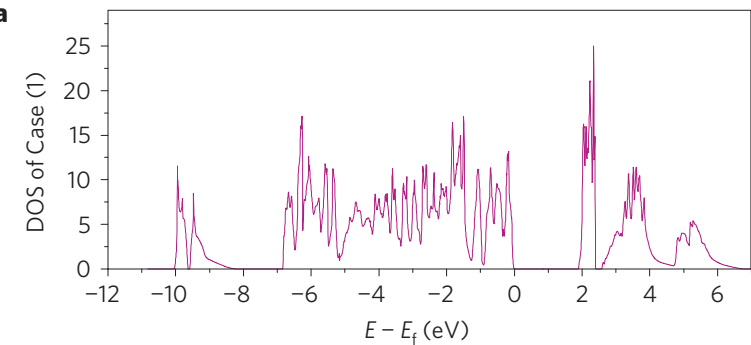

b
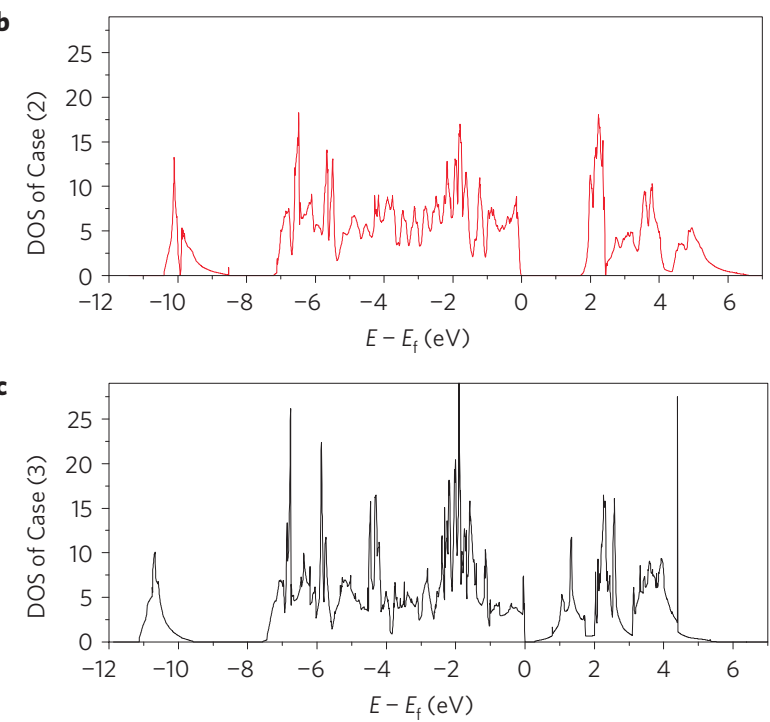

d 0.08

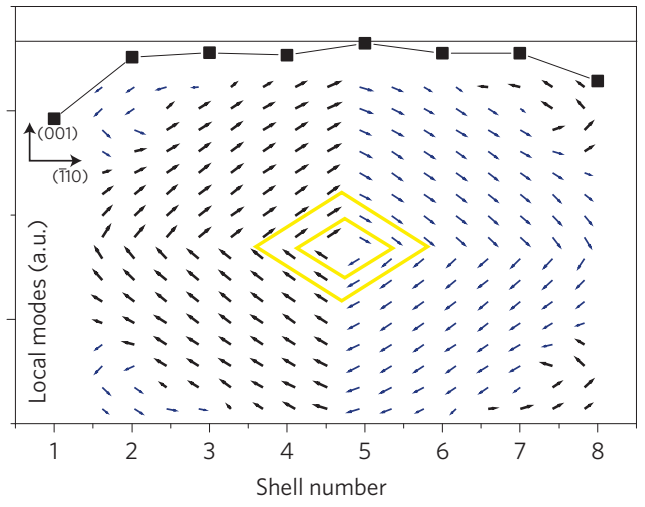

e 0.24

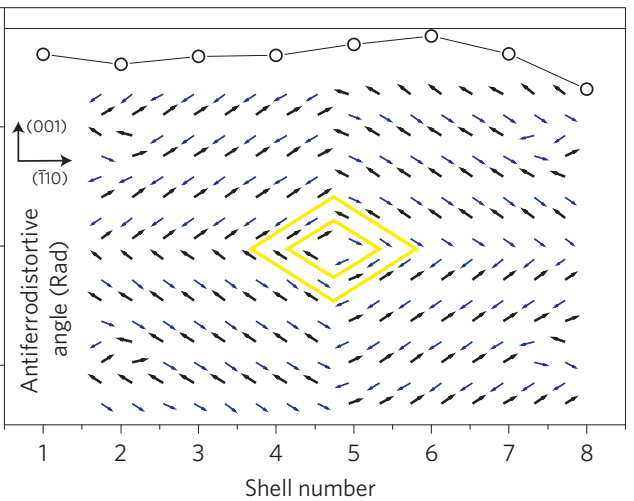

Figure 4 | DFT calculation of the electronic structure at the vortex. Comparison of the calculated density of states for a, ground-state R3c structure, b, vortex core and $\mathbf{c}$, cubic Pm $\overline{3} m$ structure. d,e, show the magnitude of the local soft modes (solid squares) and of the antiferrodistortive vectors (open circles) versus the shell number counted from the vortex core. In $\mathbf{d}$ and $\mathbf{e}$, the horizontal solid lines represent the corresponding value in the bulk. Insets of d and e show schematically the electric dipole and antiferrodistortive patterns, respectively, where only the first two shells are highlighted in the centre.

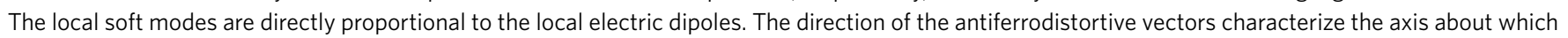
the oxygen octahedra tilt and their magnitudes provide the angle of such tilting.

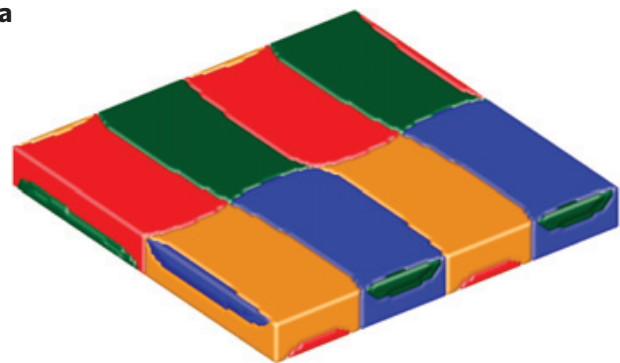

C

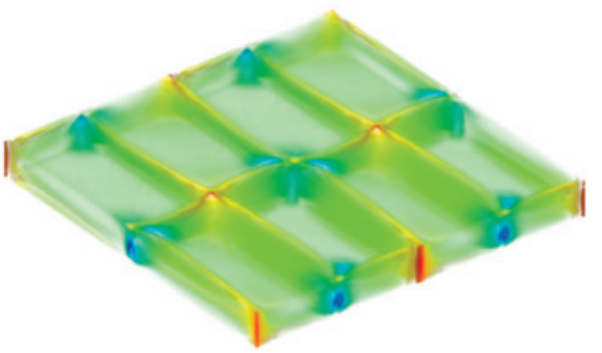

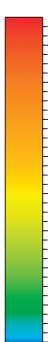
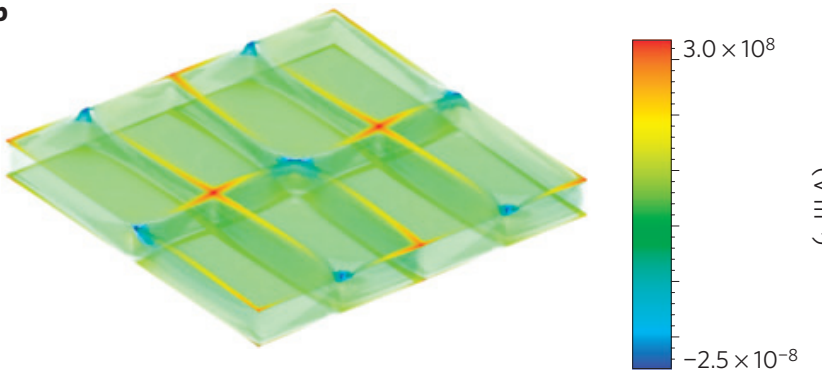

d

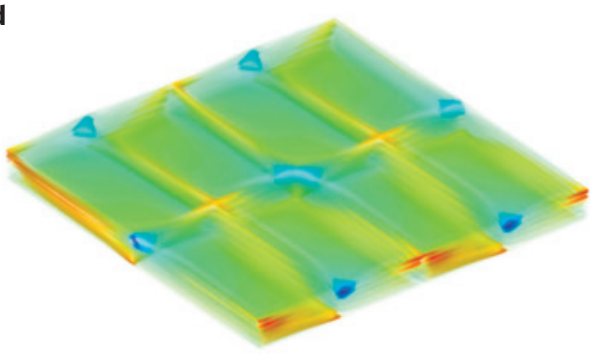

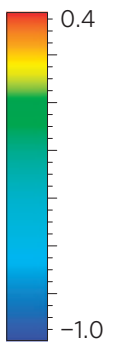

Figure 5 | Phase-field modelling of the vortex/antivortex domain arrangement. a, Domain structure, $\mathbf{b}$, electric field, $\mathbf{c}$, trace of the strain tensor, and d, potential for the vortex/antivortex lattice. Domains oriented along [1̄11] are shown in red, along [1111] in green, along [1111] in orange, and along [111] in blue.

(potential at the core versus applied potential) at the vortex and antivortex cores are the result of effective screening by polarization redistribution and the formation of charged domain walls. These charged walls, spanning the thickness of the sample, provide the conduction channels ${ }^{34}$ and are relatively insensitive to the details of the carrier segregation mechanism. 
Table 1 | Electroelastic fields at the topological defects.

\begin{tabular}{|c|c|c|c|c|c|}
\hline & $\Sigma E_{i}\left(\mathrm{Vm}^{-1}\right)$ & $\Sigma s_{i i}(\%)$ & $\Sigma \sigma_{i i}\left(\mathrm{~N} \mathrm{~m}^{-2}\right)$ & $\tilde{\beta}_{j k}^{V} \sigma_{j k}(\mathrm{eV})$ & Potential (eV) \\
\hline Vortex (m) & $3.37 \times 10^{8}$ & 1.55 & $9.75 \times 10^{9}$ & 0.174 & 0.36 \\
\hline Vortex (a) & $3.90 \times 10^{7}$ & 1.42 & $8.87 \times 10^{9}$ & 0.159 & 0.08 \\
\hline Antivortex (m) & $-2.60 \times 10^{8}$ & 0.70 & $4.35 \times 10^{9}$ & 0.078 & -1.00 \\
\hline Antivortex (a) & $-4.93 \times 10^{7}$ & -0.05 & $-2.92 \times 10^{8}$ & -0.005 & -0.31 \\
\hline Domain wall (m) & $4.16 \times 10^{7}$ & 0.35 & $2.17 \times 10^{9}$ & 0.039 & 0.17 \\
\hline Domain wall (a) & $4.07 \times 10^{7}$ & 0.18 & $1.11 \times 10^{9}$ & 0.020 & 0.04 \\
\hline Domain (a) & $8 . \times 10^{6}$ & 0.02 & $1.25 \times 10^{8}$ & 0.002 & 0.01 \\
\hline
\end{tabular}

$\Sigma E_{i}$ electric field trace, $\Sigma s_{i i}$ strain tensor trace, $\Sigma \sigma_{i i}$ stress tensor trace, $\tilde{\beta}_{j k}^{V} \sigma_{j k}$ volume expansion energy with Vegard tensor $\tilde{\beta}_{j k}^{V}$. Shown are maximum (m) and averaged (a) values.
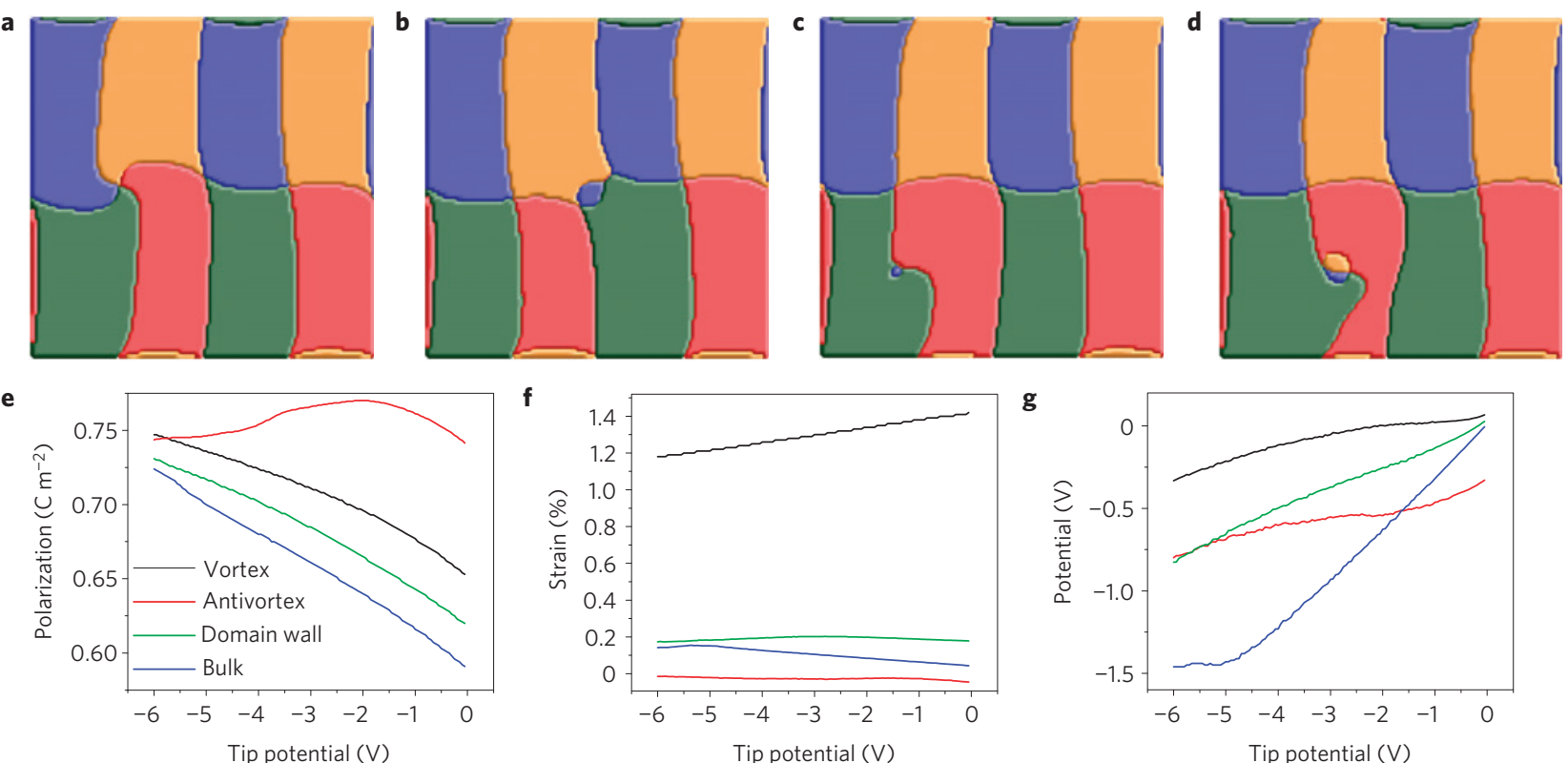

Figure 6 | Phase-field modelling of the locally biased vortex/antivortex domain arrangement. a-d, Typical domain configuration for tip-induced switching at the vortex (a), antivortex (b), domain wall (c) and domain (d) for a bias of $-6.0 \mathrm{~V}$ (top view). e-g, Evolution of electroelastic fields at the defects as a function of tip potential for average polarization (e), strain (f), and potential ( $\mathbf{g}$ ). Note that the tip potential in the simulations is negative, corresponding to

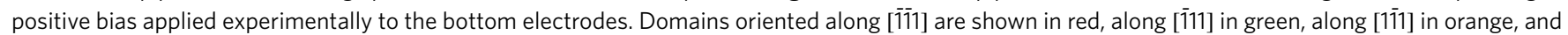
along [111] in blue.

We note that the universal aspect of the phase-field modelling is the twist of the domain walls and vortex core in the tip field ${ }^{27}$. The twist of the vortex core in modelling agrees well with the following experimental observations: (1) the c-AFM scan over the vortex structure shows localized conduction at the vortex core (Fig. 1b); (2) the conductive image over the twisted domain structure reveals a similarly looking localized conductivity (Fig. 2b) with comparable currents (compare Figs $1 \mathrm{~d}$ and $2 \mathrm{~d}$ ); (3) a rescan after (2) shows that the vortex has been restored (Fig. 2c); (4) finally, current measurements of the vortex structure sometimes do not reveal enhanced conductivity; but in these cases the rescan does not show the formation of the twist.

Briefly, we discuss the origins of the enhanced conductivity of the defect cores. We note that at the static vortex, the symmetry enhancement leads to band gap lowering (similar to domain wall ${ }^{35}$ ), estimated here to be $\sim 60-170 \mathrm{meV}$. The full relaxation of polarization in the phase-field models predicts the presence of the disclination centre (strain 0.015), favouring segregation of oxygen vacancies at the vortex cores. However, the static conductivity of the vortex and antivortex cores is expected to be different owing to the opposite (unscreened) potentials. At the same time, application of the electric bias leads to the twist of the defect cores and the formation of charged non- $71^{\circ}$ wall segments, which can lead to further accumulation of the charged electron and vacancy carriers at the defect. The twist penetrates the bulk of the material, effectively creating a 1D channel spanning between the top and bottom electrodes. The observed conductivity is thus dynamic in the sense that transport is enabled by the reversible polarization redistribution, with a corresponding critical voltage of $\sim 1 \mathrm{~V}$ as compared with $\sim 3 \mathrm{~V}$ at the wall. The back-switching of the polarization, as shown in Fig. 3f, is consistent with the redistribution of vacancies associated with the formation of a conductive channel, as (slowly relaxing) charged vacancies remaining in the off-field state result in localized polarization switching.

These studies demonstrate the controlled creation of $1 \mathrm{D}$ topological defects in ferroic materials and reveal the enhanced electronic transport along the vortex/antivortex cores. Notably, the conductance at the vortex cores is deterministic and is governed by the intrinsic material response, whereas domain wall conductivity ${ }^{36,37}$ is intrinsically linked to defect pinning and is sensitive to long-range elastic and electrostatic depolarization fields and, hence, difficult to control or predict. Vortex cores thus open a pathway for unusual states of matter which are 
stabilized at the defect cores owing to increased symmetry. We note that heterostructures of ferroelectric-ferroelastic components $^{38}$ potentially allow extending these studies towards other materials, including for example, $\mathrm{VO}_{2}$, manganites, and so on, in which metal-insulator and ferroelastic transitions are intrinsically linked.

We further note that current progress in large-scale fabrication of high-quality epitaxial films as well as advances in multiprobe $\mathrm{SPM}^{39}$ following the original Millipede concept (http:// www.zurich.ibm.com/news/05/millipede.html) suggest large-scale fabrication of topologically-ordered structures in ferroelectrics is feasible. These advances can further revitalize the areas of ferroelectric random access memories and data storage, as well other SPM-based approaches to oxide electronics ${ }^{40}$. Combinations of materials control and soft ferroic and electrochemical writing offer the potential for novel classes of electronic memory and logic devices.

\section{Methods}

Materials. The experiments were performed on $50 \mathrm{~nm}$ (001)-oriented $\mathrm{BiFeO}_{3}$ (BFO) thin films with $\mathrm{SrRuO}_{3}$ (SRO) bottom electrodes epitaxially grown on low miscut $\left(<0.01^{\circ}\right)(001) \mathrm{SrTiO}_{3}$ (STO) single crystal substrates by pulsed laser deposition $\left(670^{\circ} \mathrm{C}\right.$ at 100 mtorr of $\mathrm{O}_{2}$; ref. 41$)$. Growth was assisted by high-pressure reflection high energy electron diffraction (RHEED) analysis which indicated a layer-by-layer growth mode followed by a step flow growth mode without island formation. The heterostructures were slowly cooled to room

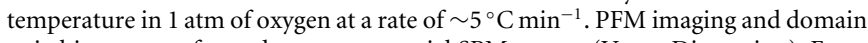
switching was performed on a commercial SPM system (Veeco Dimension). For imaging, an a.c. voltage of $2 \mathrm{~V}_{\mathrm{pp}}$ at $30 \mathrm{kHz}$ and a maximum switching voltage of $\pm 9 \mathrm{~V}$ was applied to the SPM tip during the measurement. The AFM cantilever was always oriented along the $\langle 110\rangle$ directions to maximize the in-plane PFM contrast $^{42}$. The domain structure of the as-deposited films was formed by four different domain variants, all along the [111] direction with the same out-of-plane direction pointing downwards.

Phase-field modelling. We used a phase-field model to model the evolution of the spatial polarization as a function of time, as described by the time-dependent Ginzburg-Landau equation

$$
\frac{\partial P_{i}(\mathbf{x}, t)}{\partial t}=-L \frac{\delta F}{\delta P_{i}(\mathbf{x}, t)}
$$

where $P$ is the polarization, $F$ is the sum of the Landau, elastic, electrostatic, and gradient energies, and $L$ is a kinetic parameter related to domain wall mobility. We set the initial domain structure as rectangular domains in the vortex/antivortex pattern with some thermal noise, and then allowed the system to relax until metastable.

We employed short-circuit boundary conditions for both film surfaces. When an electric bias was applied, the distribution of the electric potential on the top surface was approximated by a Lorentz distribution,

$$
\phi_{L}(x, y)=\phi_{0}\left(\frac{\gamma^{2}}{r^{2}+\gamma^{2}}\right)
$$

where $r$ is the lateral distance from the SPM tip and $\gamma$ is the half-width at half-maximum. We used a system size of $128 \Delta x \times 128 \Delta x \times 32 \Delta x$, where the thickness of the substrate and film were both $14 \Delta x$, and the substrate was allowed to elastically deform down to the bottom boundary, where it was held fixed. The data for Table 1 were taken around the defects (vortex, antivortex) and shown for the maximal value and the value averaged over the thickness. For the domain and domain wall, the values were taken away from the cores and film interfaces, at the middle of the [001]-axis of the film. We employed an isotropic gradient energy coefficient of $g_{11}=0.6$ and an isotropic dielectric constant of $\varepsilon_{i i}=50$. The Landau, elastic, and electrostrictive coefficients used are described in the literature ${ }^{43}$.

DFT calculations. We performed DFT calculations using the projector-augmented wave (PAW) formalism implemented in the Vienna Ab Initio Simulation Package (VASP; ref. 44). We applied the local spin-density approximation (LSDA) with the $+U$ Hubbard correlation method (here, $U=3.8 \mathrm{eV}$; ref. 45 ) to better treat the $3 d$ electrons of Fe. A ten-atom supercell with a G-type antiferromagnetic order was constructed to model the bulk structures discussed in the text. The plane-wave energy cutoff was set to be $500 \mathrm{eV}$. We employed a $6 \times 6 \times 6 k$-point sampling to achieve electronic convergence and the DOS was obtained by a further static calculation with a very high density of energy data points.
Received 24 May 2011; accepted 5 November 2011; published online 6 November 2011

\section{References}

1. Mermin, N. D. Topological theory of defects in ordered media. Rev. Mod. Phys. 51, 591-648 (1979).

2. Tagantsev, A. K., Cross, L. E. \& Fousek, J. Domains in Ferroelectric Crystals and Thin Films (Springer, 2010).

3. Blatter, G., Feigelman, M. V., Geshkenbein, V. B., Larkin, A. I. \& Vinokur, V. M. Vortices in high-temperature superconductors. Rev. Mod. Phys. 66, 1125-1388 (1994).

4. Ran, Y., Zhang, Y. \& Vishwanath, A. One-dimensional topologically protected modes in topological insulators with lattice dislocations. Nature Phys. 5, 298-303 (2009).

5. Salje, E. \& Zhang, H. L. Domain boundary engineering. Phase Transit. 82, 452-469 (2009).

6. Sonin, E. B. \& Tagantsev, A. K. Circulation lines and motion of antiphase walls in improper ferroelectrics. Zh. Éksp. Teor. Fiz 94, 315-328 (1988).

7. Tagantsev, A. K. \& Sonin, E. B. Linear singularities and their motion in improper ferroelectrics. Ferroelectrics 98, 297-300 (1989).

8. Sonin, E. B. \& Tagantsev, A. K. Structure and phase-transitions in antiphase boundaries of improper ferroelectrics. Ferroelectrics 98, 291-295 (1989).

9. Seidel, J. et al. Conduction at domain walls in oxide multiferroics. Nature Mater. 8, 229-234 (2009).

10. Kim, Y., Alexe, M. \& Salje, E. K. H. Nanoscale properties of thin twin walls and surface layers in piezoelectric $W_{3-x}$. Appl. Phys. Lett. 96, 032904 (2010).

11. Tselev, A. et al. Mesoscopic metal-insulator transition at ferroelastic domain walls in VO2. ACS Nano 4, 4412-4419 (2010).

12. Daraktchiev, M., Catalan, G. \& Scott, J. F. Landau theory of domain wall magnetoelectricity. Phys. Rev. B 81, 224118 (2010).

13. Salafranca, J., Yu, R. \& Dagotto, E. Conducting Jahn-Teller domain walls in undoped manganites. Phys. Rev. B 81, 245122 (2010).

14. Goncalves-Ferreira, L., Redfern, S. A. T., Artacho, E., Salje, E. \& Lee, W. T. Trapping of oxygen vacancies in the twin walls of perovskite. Phys. Rev. B 81, 024109 (2010).

15. Gruverman, A. et al. Vortex ferroelectric domains. J. Phys. Condens. Matter 20, 342201 (2008).

16. Rodriguez, B. J. et al. Vortex polarization states in nanoscale ferroelectric arrays. Nano Lett. 9, 1127-1131 (2009).

17. Ivry, Y., Chu, D. P., Scott, J. F. \& Durkan, C. Flux closure vortexlike domain structures in ferroelectric thin films. Phys. Rev. Lett. 104, 207602 (2010).

18. McGilly, L. J., Schilling, A. \& Gregg, J. M. Domain bundle boundaries in single crystal $\mathrm{BaTiO}_{3}$ lamellae: Searching for naturally forming dipole flux-closure/quadrupole chains. Nano Lett. 10, 4200-4205 (2010).

19. $\mathrm{Wu}, \mathrm{Z}$. et al. Unusual vortex structure in ultrathin $\mathrm{Pb}\left(\mathrm{Zr}_{0.5} \mathrm{Ti}_{0.5}\right) \mathrm{O}_{3}$ films. J. Appl. Phys. 101, 014112 (2007).

20. Naumov, I. \& Fu, H. Vortex-to-polarization phase transformation path in ferroelectric $\mathrm{Pb}(\mathrm{ZrTi}) \mathrm{O}_{3}$ nanoparticles. Phys. Rev. Lett. 98, 077603 (2007).

21. Slutsker, J., Artemev, A. \& Roytburd, A. Phase-field modeling of domain structure of confined nanoferroelectrics. Phys. Rev. Lett. 100, 087602 (2008).

22. Prosandeev, S., Ponomareva, I., Naumov, I., Kornev, I. \& Bellaiche, L. Original properties of dipole vortices in zero-dimensional ferroelectrics. J. Phys. Condens. Matter 20, 193201 (2008).

23. Wang, J. Switching mechanism of polarization vortex in single-crystal ferroelectric nanodots. Appl. Phys. Lett. 97, 192901 (2010).

24. Jia, C-L., Urban, K. W., Alexe, M., Hesse, D. \& Vrejoiu, I. Direct observation of continuous electric dipole rotation in flux-closure domains in ferroelectric $\mathrm{Pb}(\mathrm{Zr}, \mathrm{Ti}) \mathrm{O}_{3}$. Science 331, 1420-1423 (2011).

25. Nelson, C. T. et al. Spontaneous vortex nanodomain arrays at ferroelectric heterointerfaces. Nano Lett. 11, 828-834 (2011).

26. McQuaid, R. G. P., McGilly, L. J., Sharma, P., Gruverman, A. \& Gregg, J. M. Mesoscale flux-closure domain formation in single-crystal $\mathrm{BaTiO}_{3}$. Nature Commun. 2, 404 (2011).

27. Balke, N. et al. Deterministic control of ferroelastic switching in multiferroic materials. Nature Nanotechnol. 4, 868-875 (2009).

28. Maksymovych, P. et al. Polarization control of electron tunneling into ferroelectric surfaces. Science 324, 1421-1425 (2009).

29. Jesse, S., Kalinin, S. V., Proksch, R., Baddorf, A. P. \& Rodriguez, B. J. The band excitation method in scanning probe microscopy for rapid mapping of energy dissipation on the nanoscale. Nanotechnology 18, 435503 (2007).

30. Buhlmann, S., Colla, E. \& Muralt, P. Polarization reversal due to charge injection in ferroelectric films. Phys. Rev. B 72, 214120 (2005).

31. Kholkin, A. L., Bdikin, I. K., Shvartsman, V. V. \& Pertsev, N. A. Anomalous polarization inversion in ferroelectrics via scanning force microscopy. Nanotechnology 18, 095502 (2007).

32. Sichuga, D., Ren, W., Prosandeev, S. \& Bellaiche, L. Chiral patterns of tilting of oxygen octahedra in zero-dimensional ferroelectrics and multiferroics: A first principle-based study. Phys. Rev. Lett. 104, 207603 (2010). 
33. Neaton, J. B., Ederer, C., Waghmare, U. V., Spaldin, N. A. \& Rabe, K. M. First-principles study of spontaneous polarization in multiferroic $\mathrm{BiFeO}_{3}$. Phys. Rev. B 71, 014113 (2005).

34. Eliseev, E. A., Morozovska, A. N., Svechnikov, G. S., Gopalan, V. \& Ya. Shur, V. Static conductivity of charged domain wall in uniaxial ferroelectric-semiconductors. Phys. Rev. B 83, 235313 (2011).

35. Catalan, G. \& Scott, J. F. Physics and applications of bismuth ferrite. Adv. Mater. 21, 2463-2485 (2009).

36. Maksymovych, P. et al. Dynamic conductivity of ferroelectric domain walls in $\mathrm{BiFeO}_{3}$. Nano Lett. 11, 1906-1912 (2011).

37. Farokhipoor, S. \& Noheda, B. Conduction through $71^{\circ}$ domain walls in $\mathrm{BiFeO}_{3}$ thin films. Phys. Rev. Lett. 107, 127601 (2011).

38. Biegalski, M. D., Doerr, K., Kim, D. H. \& Christen, H. M. Applying uniform reversible strain to epitaxial oxide films. Appl. Phys. Lett. 96, 151905 (2010).

39. Rosenwaks, Y., Dahan, D., Molotskii, M. \& Rosenman, G. Ferroelectric domain engineering using atomic force microscopy tip arrays in the domain breakdown regime. Appl. Phys. Lett. 86, 012909 (2005).

40. Cen, C., Thiel, S., Mannhart, J. \& Levy, J. Oxide nanoelectronics on demand. Science 323, 1026-1030 (2009).

41. Martin, L. W. et al. Nanoscale control of exchange bias with $\mathrm{BiFeO}_{3}$ thin films. Nano Lett. 8, 2050-2055 (2008).

42. Zavaliche, F. et al. Ferroelectric domain structure in epitaxial $\mathrm{BiFeO}_{3}$ films. Appl. Phys. Lett. 87, 182912 (2005).

43. Zhang, J. X., Schlom, D. G., Chen, L. Q. \& Eom, C. B. Tuning the remanent polarization of epitaxial ferroelectric thin films with strain. Appl. Phys. Lett. 95, 122904 (2009).

44. Kresse, G. \& Furthmüller, J. Efficiency of $a b$-initio total energy calculations for metals and semiconductors using a plane-wave basis set. Comput. Mater. Sci. 6 15-50 (1996).

45. Kornev, I., Lisenkov, S., Haumont, R., Dkhil, B. \& Bellaiche, L. Finite-temperature properties of multiferroic $\mathrm{BiFeO}_{3}$. Phys. Rev. Lett. 99, 227602 (2007).

\section{Acknowledgements}

Experiments were conducted at the Center for Nanophase Materials Sciences, which is sponsored at Oak Ridge National Laboratory by the Office of Basic Energy Sciences, US Department of Energy. Support was provided by the Division of Scientific User Facilities (N.B.) and by the Materials Sciences and Engineering Division (S.V.K.) of the US Department of Energy, Basic Energy Sciences. B.W., J.B. and L.Q.C. are supported by US Department of Energy, Basic Sciences, under Grant No. DE-FG02-07ER46417. L.B. thanks mostly support from the Department of Energy, Office of Basic Energy Sciences, under contract ER-46612. L.B. also thanks the National Science Foundation grants DMR-1066158 and DMR-0701558, and Office of Naval Research grants N00014-11-1-0384, N00014-08-1-0915 and N00014-07-1-0825. Some computations were also made possible thanks to the National Science Foundation grant 0722625 and a challenge grant from the US Department of Defense. Y.H.C. acknowledges the support of the National Science Council, Republic of China, under contract NSC-100-2811-M-009-003. M.H. acknowledges support by the Netherlands Organization for Scientific Research (NWO) through a VENI grant.

\section{Author contributions}

N.B. and S.V.K. conceived and designed the experiments, and wrote the article. N.B performed the experiments. B.W., J.B. and L.Q.C. performed phase-field modelling. W.R., L.B. and I.K. performed DFT calculations. A.N.M. and E.A.E. provided analytical theory for vortex structure and vacancy segregation. M.H., Y.H.C. and R.R. contributed materials and S.J. developed spectroscopic measurement technique and analysis tools. S.V.K., P.M. and R.K.V. co-wrote the article. All authors discussed the results and commented on the manuscript.

\section{Additional information}

The authors declare no competing financial interests. Supplementary information accompanies this paper on www.nature.com/naturephysics. Reprints and permissions information is available online at http://www.nature.com/reprints. Correspondence and requests for materials should be addressed to N.B. or S.V.K. 